\title{
Varying the effective mass of geophones
}

\author{
K. T. Spikes*, D. W. Steeples*, C. M. Schmeissner ${ }^{\ddagger}$, \\ R. Prado**, and M. Pavlovic ${ }^{\S}$
}

\begin{abstract}
Traditionally, acquiring seismic data has rested on the assumption that geophone mass should be as small as possible. When Steeples and coworkers in 1999 planted 72 geophones automatically and simultaneously with a farm tillage implement, the effective mass of each of the geophones was significantly increased. We examined how the mass of a geophone affects changes in traveltime, amplitude, frequency, and overall data quality by placing various external masses on top of $100-\mathrm{Hz}$ vertical geophones. Circular barbell weights of 1.1-, 11.3-, and $22.7 \mathrm{~kg}$; an $8.2-\mathrm{kg}$ bag of lead shot; and a $136-\mathrm{kg}$ stack of barbell weights were placed on top of geophones during data acquisition. In addition, a very large mass in the form of a truck was parked on top of two of the geophones. Four seismic sources supplying a broad range of energies were tested: a sledgehammer, a .22-caliber rifle, a 30.06 rifle, and an 8-gauge Betsy Seisgun. Spectral analysis revealed that the smaller weights had the greatest effects on the capacities of the geophones to replicate the earth's motion. Consequently, using geophones with a large effective mass as part of an automatic geophoneplanting device would not necessarily be detrimental to the collection of high-quality near-surface seismic data.
\end{abstract}

\section{INTRODUCTION}

One assumption made in reflection seismology has been that the mass of the wave sensor does not significantly affect its ability to oscillate at the same time and frequency as the earth beneath it. Although experimental and theoretical results have been obtained using massive geophones (Wolf, 1944; Hoover and O'Brien,1980), the effect of a large range of masses on geophone oscillation has not been fully explored. Steeples et al. (1999) reintroduced the massive geophone issue while discussing possible automatic geophone-planting devices. In their experiments, 72 geophones were attached to a hydraulically powered farm-tillage tool and then planted automatically and simultaneously. The steel frame of the implement and the iron bars to which the geophones were attached added substantial effective mass to each receiver. The experiments involving the farm implement were not designed to examine the effects of geophone mass on receiver response. Therefore, the purpose of the experiments discussed here was to test how geophone mass affected the traveltime, amplitude, and frequency of shallow subsurface seismic data.

Previous work on this subject includes that of Wolf (1944), who showed theoretically that a geophone resting on an elastic surface reacted to incident elastic waves like a simple, damped harmonic oscillator. He assumed that the geophone was a right cylinder with a mass of approximately $11.3 \mathrm{~kg}$ and that the circular base plate was in perfect contact with the ground. Under these assumptions and by solving the second-order linear differential equations of motion, Wolf showed that the mass of the geophone affected its ability to replicate the movement of the earth. An absolute minimum mass, theoretically zero, was needed to duplicate broadband ground motion. He determined that the ground motion due to frequencies above the natural frequency of the oscillating system would not be faithfully reproduced but that geophones could replicate the ground motions associated with lower frequencies (Wolf, 1944).

Hoover and O'Brien (1980) experimented with geophones equipped with circular base plates. Assuming a semi-infinite, elastic earth, they concluded that the frequency at which the geophone oscillated was a function of its mass and the size of the base plate. When the mass was small and the base plate

Presented at the 70th Annual International Meeting, Society of Exploration Geophysicists. Manuscript received by the Editor March 8, 2000; revised manuscript received January 19, 2001.

*University of Kansas, Department of Geology, 120 Lindley Hall, Lawrence, Kansas 66045-2124. E-mail: kpilot@ukans.edu; dsteeples@ukans.edu. $\ddagger$ Formerly University of Kansas, Department of Geology, 120 Lindley Hall, Lawrence, Kansas 66045; presently Jefferson County GIS, P. O. Box 447, Oskaloosa, Kansas 66066. E-mail: gis@ruralnet1.com.

** Formerly University of Kansas, Department of Geology, 120 Lindley Hall, Lawrence, Kansas 66045; presently Institute for Technological Research, 532 Prof. Almeida Prado Ave., Sao Paulo, Brazil. E-mail: prado@ipt.br.

${ }^{\S}$ Formerly University of Kansas, Department of Electrical Engineering and Computer Science, Center for Research, Lawrence, Kansas 66047; presently Boeing Satellite Systems, Inc., P. O. Box 92919, Los Angeles, California 90009-2919. E-mail: mario.pavlovic@being.com.

(C) 2001 Society of Exploration Geophysicists. All rights reserved. 
relatively large, a large amount of damping of the geophone motion occurred. A smaller base plate and a heavier mass exhibited low resonant frequencies (Hoover and O'Brien, 1980). Krohn (1984) conducted additional experimental analysis using modern, lightweight, spiked geophones. She concluded that accurate data collection does not depend on the mass of the geophone and its replication of the earth's motion, but on the quality of the coupling of the geophone to the ground, which is a direct result of the firmness or solidity of the soil (Krohn, 1984).

Drijkoningen (2000) further discussed geophone-ground coupling by noting two types: spike-shear coupling and weight coupling. For spike-shear coupling, a spiked geophone is coupled to the ground by friction between the spike and the ground, and the geophone plant is considered to be well coupled. Weight coupling refers only to the weight of the geophone that furnishes the geophone-ground coupling, and the geophone is not considered to be well coupled to the ground. Although the weight of the geophone as a coupling mechanism is discussed, the effect of weight on geophone performance is not mentioned. No definitive answer to the geophone-mass issue was obtained. Most notably, no experimental results appear to have been published in which spiked geophones, highly variable masses, and identical geophone elements were tested.

\section{METHODS}

Four data sets were acquired in a small grass-covered field near the Kansas Biological Survey at the University of Kansas in Lawrence, Kansas. Underlying a clay-rich soil at this site are alternating limestones and shales in the Lawrence formation of Pennsylvanian age (O'Connor, 1960). Soil-moisture conditions are known to be important to data quality at this site (Jefferson et al., 1998). Therefore, data were collected four times during the months of June through August, 1999, to assure variations in surface soil-moisture conditions that could otherwise bias results. The first data set was acquired on June 18, when soilmoisture conditions allowed the field crew to plant geophones easily (i.e., without having to step on them to force them into the ground). Approximately $10 \mathrm{~cm}$ of rain had fallen four days prior to data collection. On June 30, the second set of data was recorded. Two days before, enough rain had fallen to saturate the ground completely. The third data set was collected July 14 , when soil conditions were drier than on June 18. However, some moisture remained in the ground. On August 25, the fourth data set was taken. No significant rainfall had occurred in the previous six weeks, which left the ground dry and hard.

To examine how the mass of a geophone affects changes in traveltime, amplitude, frequency, and overall data quality, various external masses were balanced on the tops of $100-\mathrm{Hz}$ vertical geophones. The four data sets were recorded under varying soil-moisture conditions with equivalent field parameters, but the geophones were replanted each time. Forty-eight $100-\mathrm{Hz}$ Mark Products L-40A vertical geophones with $12.5-\mathrm{cm}$ spikes were placed at spacings of $0.5 \mathrm{~m}$. During data acquisition, 1.1-, $11.3-$, and $22.7-\mathrm{kg}$ circular barbell weights, an $8.2-\mathrm{kg}$ bag of lead shot, and a truck representing a very large mass were placed on designated geophones (Figure 1). The barbell weights and the bag of lead shot were placed atop separate geophones at specific shot-to-geophone offsets.

The wheels of the truck were used to perform two tasks. First, one of the dual wheels from each side of the truck was driven onto and then off a geophone, thus pressing it into the ground about $2-3 \mathrm{~cm}$. The truck was then moved $0.5 \mathrm{~m}$ along the line. For the second experiment with the truck, one dual wheel from each side of the truck was parked on a geophone during acquisition. Each dual truck wheel added approximately $1000 \mathrm{~kg}$ of mass in the vicinity of the geophone. Of that $1000 \mathrm{~kg}$, at least $115 \mathrm{~kg}$ of mass were added to each of the two geophones, assuming $551600 \mathrm{~Pa}$ of internal tire air pressure and approximately $2000 \mathrm{~mm}^{2}$ of tire in contact with the geophone. Lastly, an experiment was performed in which a 136-kg stack of barbell weights was balanced atop one geophone. The 136-kg stack of barbell weighs was used to add to the geophone a mass similar to that contributed directly by the truck.

Four sources supplying a broad range of energies were tested: a sledgehammer, a .22-caliber rifle, a 30.06 rifle, and an 8-gauge Betsy Seisgun, with the latter two yielding similar results. Shots were taken in-line, one source at a time, at $1-, 25-$, and 49-m offsets from the nearest geophone, where the shot increment equaled the receiver line length and the receivers remained at fixed locations. The .22-caliber rifle firing supersonic, long-rifle ammunition produced the highest frequencies but provided little usable energy at the far offsets. When the sledgehammer was used to strike an aluminum plate, five impacts were recorded individually at each location to be stacked during processing. The sledgehammer did not produce consistent results in the four data sets, partly because of the difficulty of striking the impact plate consistently with regard to strength and location (Keiswetter and Steeples, 1995). The 30.06 rifle and the 8-gauge Betsy Seisgun provided the most consistent and coherent data, with the Betsy Seisgun producing the most coherent results associated with increased effective geophone mass. Thus, the following presentation centers on the data acquired using the Betsy Seisgun as the source.

Changes in traveltime, amplitude, and frequency were determined by comparing the traces corresponding to weighted geophones with adjacent traces, which corresponded to unweighted geophones. Amplitude and frequency analysis were done on raw data. Traveltime variations were established using a bandpass filter with a $150-\mathrm{Hz}$ low-cut and a $400-\mathrm{Hz}$ high-cut filter, a $12 \mathrm{~dB} /$ octave roll-off on both ends, and a $6-\mathrm{dB}$ linear gain but no automatic gain control (Figures 2, 3).

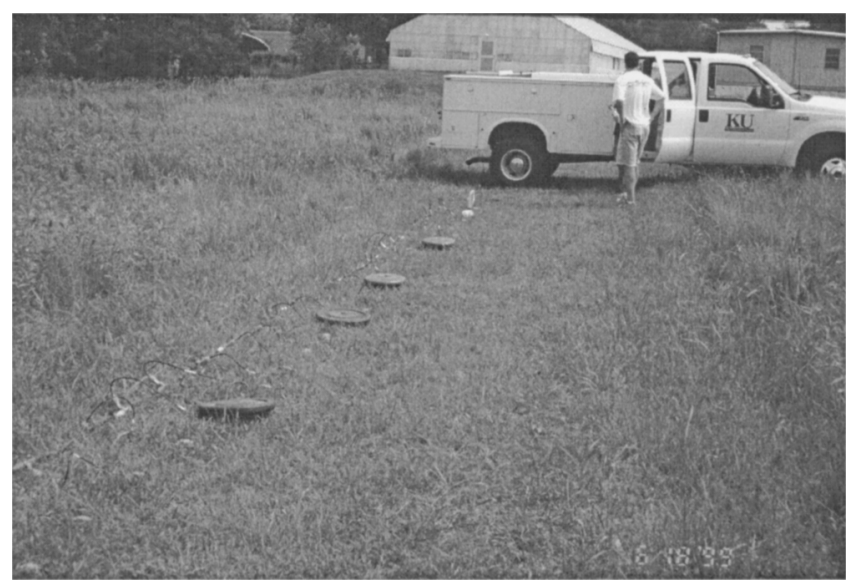

FIG. 1. The various weights balanced on the tops of the $100-\mathrm{Hz}$ vertical geophones include (from front to back) two $22.7-\mathrm{kg}$ and two 11.3-kg circular barbell weights, an $8.2-\mathrm{kg}$ bag of lead shot, a truck, and two 1.1-kg barbell weights (behind the truck). 
Two procedures were conducted before frequency filtering to analyze the amplitude changes in the traces associated with the weights. First, trace-to-trace plots were compared. Individual traces corresponding to the different weighted geophones and adjacent traces from the unweighted geophones were plotted and compared visually (Figure 4). For each data set, the geophone interval was $0.5 \mathrm{~m}$. Comparing traces corresponding to two adjacent, unweighted geophones showed no significant differences in the amplitudes. Therefore, any amplitude differences between the weighted and unweighted geophones caused by the 0.5 -m offset differences were not thought to be significant.

Second, the rms amplitudes of the peaks of the first-arrival refraction wavelets and the $\sim 80$-ms two-way traveltime reflection wavelets were determined in the traces corresponding to the weighted geophones and the adjacent traces with the unweighted geophones. In addition, the rms amplitude of the early noise prior to the first arrival was found in both types of traces. Amplitude S/Ns were calculated by dividing both the refraction and the reflection signal amplitudes by the noise am-

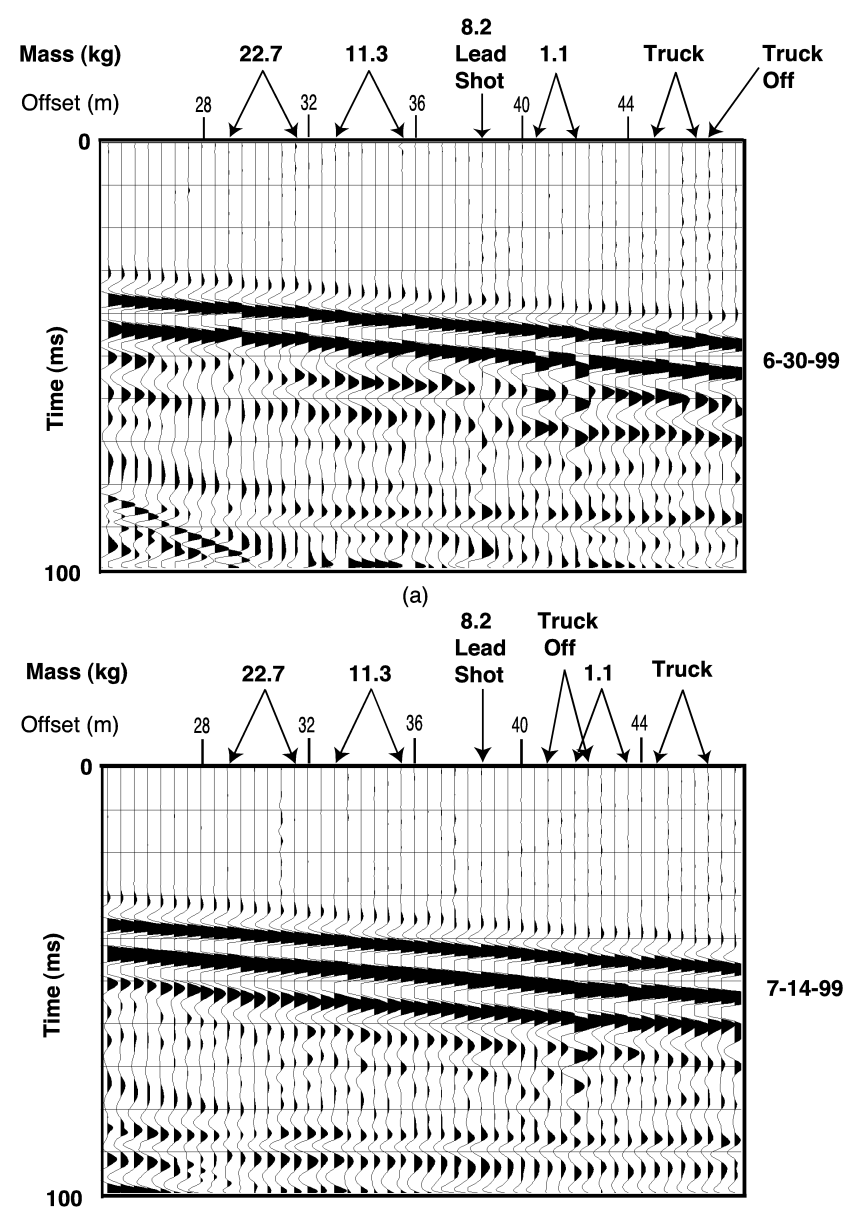

(b)

FIG. 2. (a) Record acquired June 30, 1999, under saturated surface-soil conditions showing traveltime differences in the weighted-geophone traces. (b) Drier surface-soil conditions such as those on August 14, 1999, yielded traveltime shifts in weighted-geophone traces different from those that occurred under the saturated surface-soil conditions associated with (a). Both records are displayed with a $150-400 \mathrm{~Hz}$ bandpass filter and $6-\mathrm{dB}$ linear gain. plitude. Subtracting the ratio of an unweighted geophone from the ratio corresponding to a weighted, adjacent geophone gave the $\mathrm{S} / \mathrm{N}$ difference. To show the $\mathrm{S} / \mathrm{N}$ difference with increased effective mass, a plot was constructed showing the increased mass versus $\mathrm{S} / \mathrm{N}$ change (Figures $5 \mathrm{a}, \mathrm{b}$ ). A positive change in $\mathrm{S} / \mathrm{N}$ denotes an increase in the ratio, whereas a negative change is a decrease in $\mathrm{S} / \mathrm{N}$.

The data plotted in Figure 5 shows that varying results were obtained when using the same masses on geophones. The sources of these variations were not examined but could include local variation in soil makeup, geophone plants that were not exactly vertical, and buried roots from brush that had been growing in the field previously, among others.

The loss in frequency content was found by comparing the frequency spectra of the individual traces corresponding to a weighted geophone against the individual frequency spectra of traces from the adjacent, unweighted geophones (Figure 6). Frequency content was examined from data collected at all three source offsets. The primary range of frequencies was from 150 to $400 \mathrm{~Hz}$.

\section{RESULTS}

Geophone response was affected by effective mass in the form of a geophone-barbell weight system, but a truck and a $136-\mathrm{kg}$ stack of barbell weights did not affect geophone response nearly as much as did some of the smaller masses. This result was unexpected because the truck and the stack of weights were significantly heavier than the geophone-barbell weight systems.

Frequency-filtered data showed no visible traveltime shifts in the first arrivals in the 1.1-kg weighted geophone traces under both saturated and dry conditions. However, after the first arrivals, these traces displayed a high-amplitude ringing throughout the remainder of the traces; all later events revealed 1-2 ms
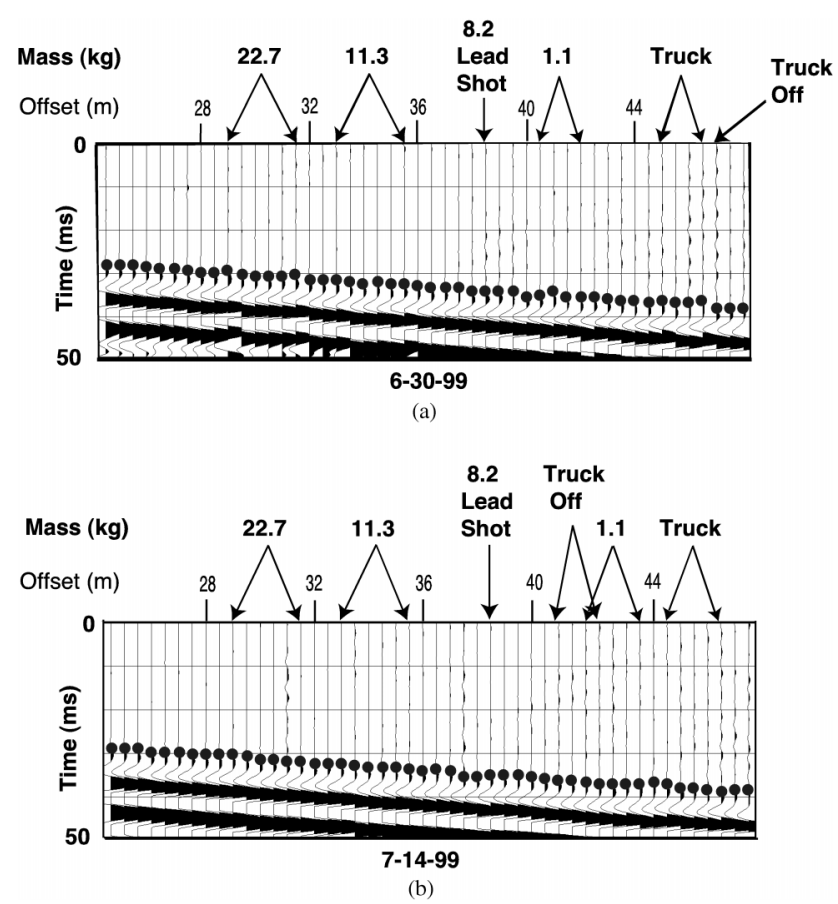

FIG. 3. Plots of first arrival picks with mass annotated from (a) June 30 and (b) August 14 
time delays (Figures 2, 3). This ringing, which does not show up as an obvious feature in the corresponding amplitude spectra, could be attributed to the $150-\mathrm{Hz}$ low-cut filter that was applied in the time domain but was not applied to the data used to calculate the amplitude spectrum. The traces corresponding to other geophones with small masses on their tops did not ring visibly; therefore, the 1.1-kg mass may have introduced conditions near resonance for the geophone-plant system.

Under saturated conditions, the $11.3-$ and $22.7-\mathrm{kg}$ masses caused early first arrivals of $1 \mathrm{~ms}$ or less (Figures 2a, 3a). Under drier conditions, the 11.3- and 22.7-kg masses caused no visible traveltime shifts (Figures $2 b, 3 b$ ). Traveltime delays were present in events after the first arrivals under saturated conditions in the lead-shot-weighted geophone traces, but no traveltime differences were seen when soil conditions were drier (Figures 2,3). Traces from the geophones on which the truck (Figures 2,3) and the 136-kg stack of weights were placed displayed 1-ms early first arrivals. No visible traveltime shifts relative to the adjacent traces were present in the traces corresponding to the geophones from which the weight of the truck had been removed (Figures 2, 3). (a)

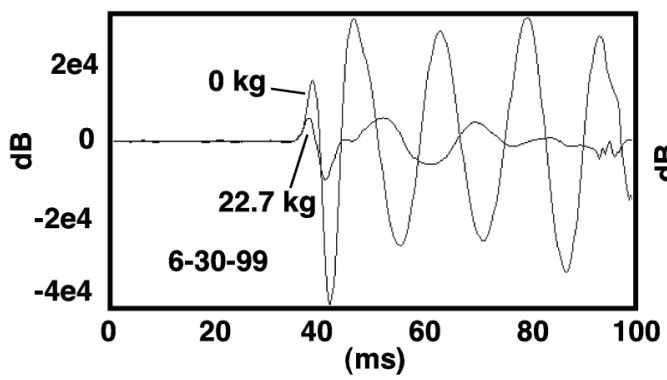

(c)

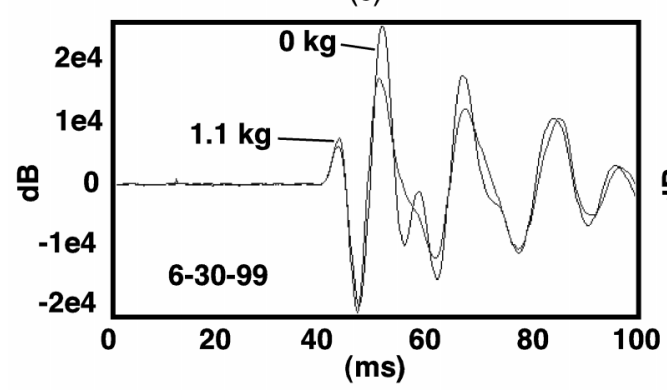

(e)

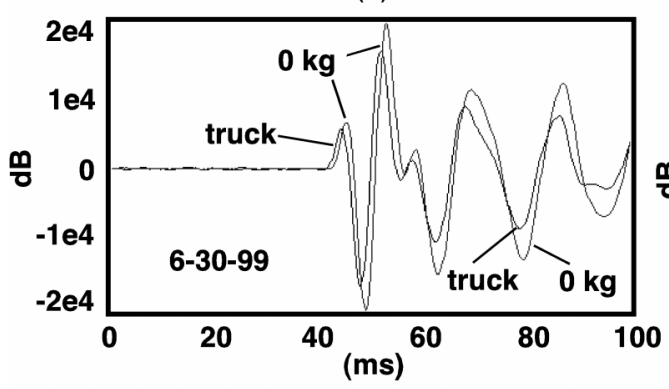

(g)

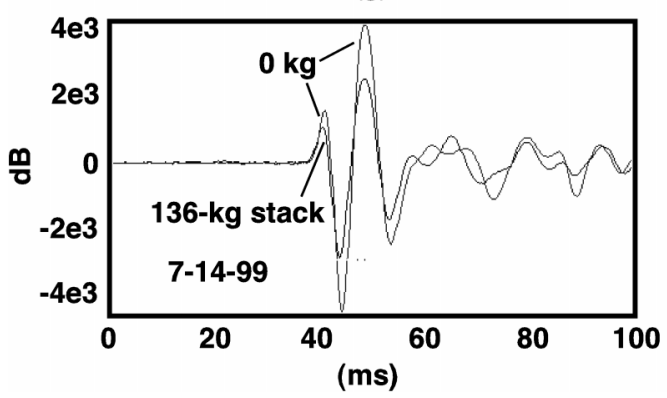

(b)

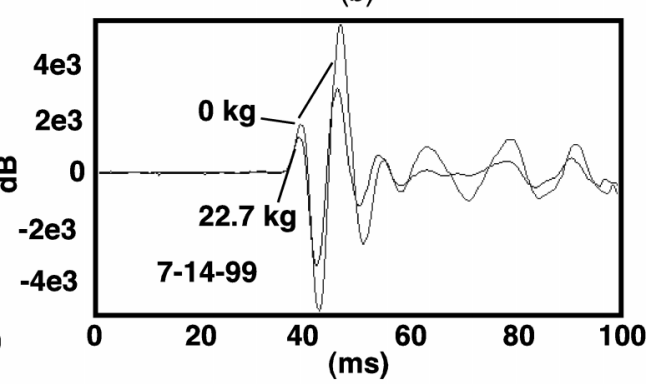

(d)
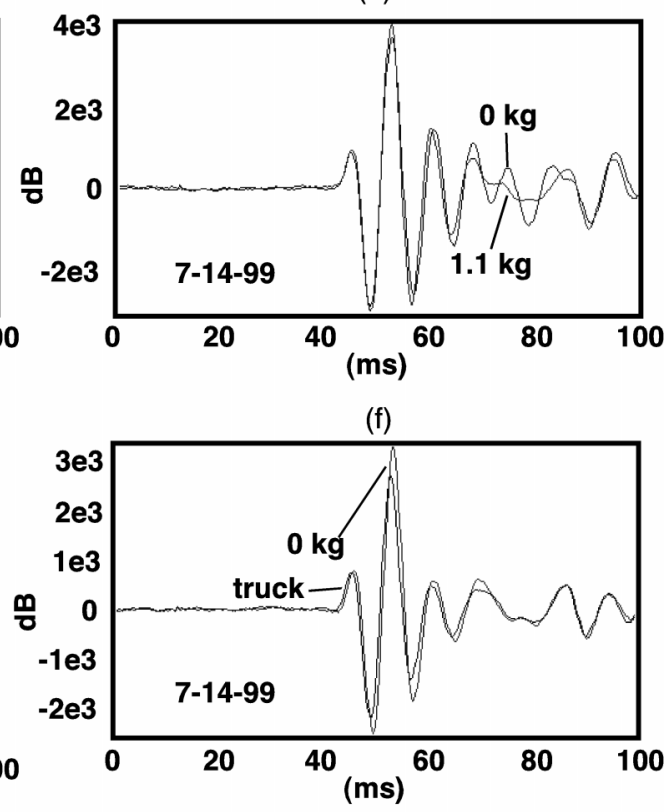
Direct trace-to-trace plot comparisons yielded slightly different results than the amplitude $\mathrm{S} / \mathrm{N}$ comparisons. Comparing a trace corresponding to a $22.7-\mathrm{kg}$ weight and an adjacent, unweighted geophone trace, showed that the $22.7-\mathrm{kg}$ weight decreased the amplitude throughout the trace in both saturated and dry soil conditions (Figures 4a, b). Similar comparisons with a 1.1-kg weight yielded much smaller amounts of amplitude loss, particularly with the first-arrival refraction and the $\sim 80$-ms reflection (Figures $4 \mathrm{c}, \mathrm{d}$ ). In saturated conditions, the first-break amplitude in a trace from a truck-weighted geophone remained unchanged, but the $\sim 80$-ms reflection amplitude decreased by $\sim 2 \mathrm{~dB}$ relative to an adjacent trace (Figure $4 \mathrm{e}$ ). Under dry conditions, virtually no amplitude was lost in either the first break or the $\sim 80$-ms reflection relative to an unweighted-geophone trace (Figure 4f). Lastly, comparing the geophone trace corresponding to the stack of barbell weights to an unweighted-geophone trace revealed that the stack of weights caused a larger decrease in amplitude than the truck did in the first arrival and the $\sim 80$-ms reflection (Figure $4 \mathrm{~g}$ ).

Amplitude S/N comparisons showed that the 136-kg stack of barbell weights caused the most significant drop in S/N (Figures 5a, b). The smaller barbell weights and the bag of lead shot did not reduce $\mathrm{S} / \mathrm{N}$ as significantly as did the stack of weights. In the truck-weighted geophone traces, $\mathrm{S} / \mathrm{N}$ was preserved or reduced by a relatively small amount $(5 \mathrm{~dB})$. Moreover, the amount of amplitude $\mathrm{S} / \mathrm{N}$ lost in the truck-weighted geophone traces was similar to the amount lost in the $1.1-\mathrm{kg}$ weightedgeophone traces $(2 \mathrm{~dB})$. Virtually no $\mathrm{S} / \mathrm{N}$ was lost in the traces corresponding to the geophones from which the truck's weight had been removed (Figures 5a, b). Similar results were obtained in both dry and saturated conditions. Although traceto-trace plots showed that amplitudes decreased (Figure 4), the $\mathrm{S} / \mathrm{N}$ increased, in most cases, up to $25 \mathrm{~kg}$ of increased effective mass (Figure 5).

The frequency spectra from unfiltered traces demonstrated that the most significant loss of amplitude $(5-15 \mathrm{~dB})$ in the 150 $400-\mathrm{Hz}$ range was due to the $1.1-\mathrm{kg}$ weights in both dry and saturated soil conditions (Figures $6 \mathrm{a}, \mathrm{b}$ ). In both soil conditions, the 11.3- and 22.7-kg weights and the bag of lead shot produced amplitude losses of about $5 \mathrm{~dB}$ in this frequency range. The frequency content and amplitude of the truck-weighted geophone traces was approximately the same as for the adjacent traces in both dry and saturated soil conditions (Figures $6 c, d$ ). No significant amount of frequency content in the 150-400-Hz range was lost due to the stack of weights (Figure 6e) or to the truck's weight being driven onto and then off a geophone.

The geophone-truck system differs from the geophonebarbell system in that the geophone is in contact with rubber, which is less rigid than iron. However, a heavy-duty truck tire and an iron barbell weight may appear more similar to a seismic $P$-wave traveling a few hundred meters per second than would be expected intuitively. Hence, we believe that the degree of elasticity of the rubber tires is not an important factor in our analysis. Alternatively, the data acquired using the two systems could be regarded as having resulted from two separate experiments.

Lastly, Drijkoningen (2000) indicated that a geophone coupled to the ground primarily by its weight is not well coupled. The mass of our most heavily weighted geophones assisted in coupling them to the ground, and this coupling appeared to help, not hinder, geophone performance.

\section{CONCLUSIONS}

When highly variable masses were added to geophones and their responses to the masses were examined, the effect of increasing the effective mass greatly did not appear to be detrimental to the acquisition of high-quality near-surface seismic data. The geophone-barbell system increased the effective mass of the geophone and hindered geophone performance in terms of amplitude. The geophone-truck system increased the effective mass of the geophone enough that the $P$-wave velocity of the near surface surrounding the geophone increased also, and geophone performance was hindered less. Although the smaller masses such as the barbell weights and the bag of lead shot decreased the amplitude and frequency of the signal, $\mathrm{S} / \mathrm{N}$ increased in most cases with these masses. However, the 136-kg stack of barbell weights caused a drop in amplitude, frequency, and $\mathrm{S} / \mathrm{N}$. The truck did not significantly

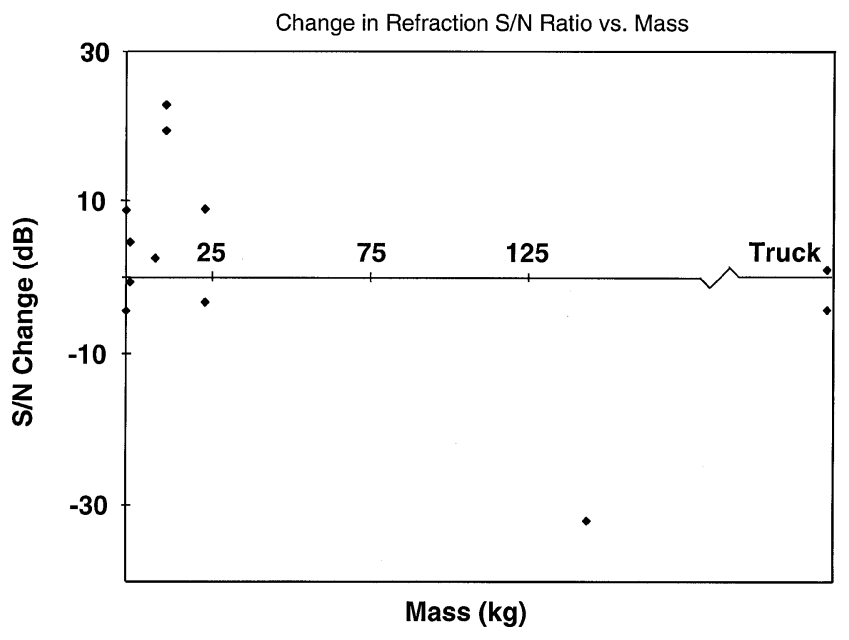

(a)

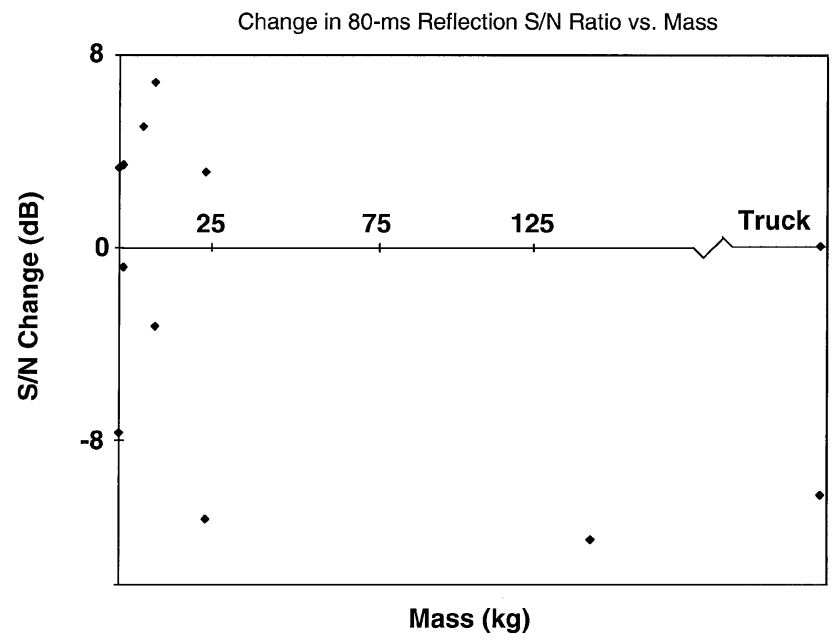

(b)

FIG. 5. (a) The change in the first refraction $\mathrm{S} / \mathrm{N}$ of unweightedto weighted-geophone traces when plotted versus the added mass from August 14 shows a significant loss due to the $136-\mathrm{kg}$ stack of barbell weights. However, for the smaller masses and the mass of the truck, the ratio either increases or slightly decreases. (b) This similar plot uses the $\sim 80$-ms reflection amplitude. The S/N decreased with increasing mass. 


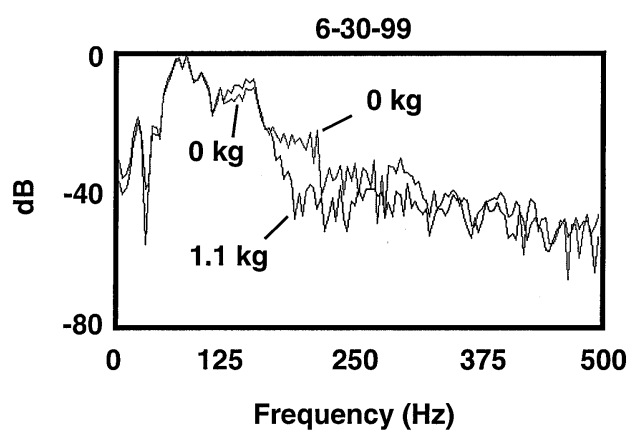

(a)

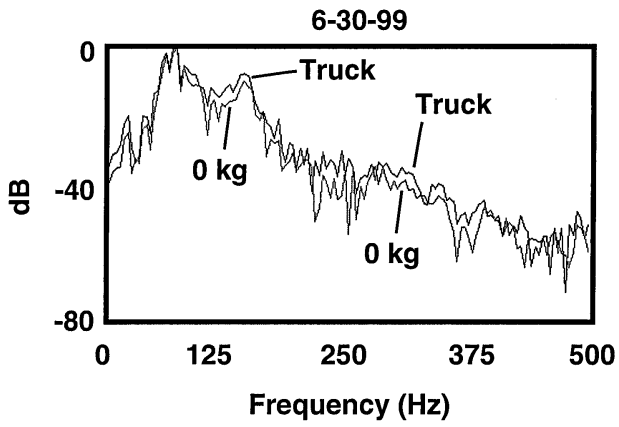

(c)

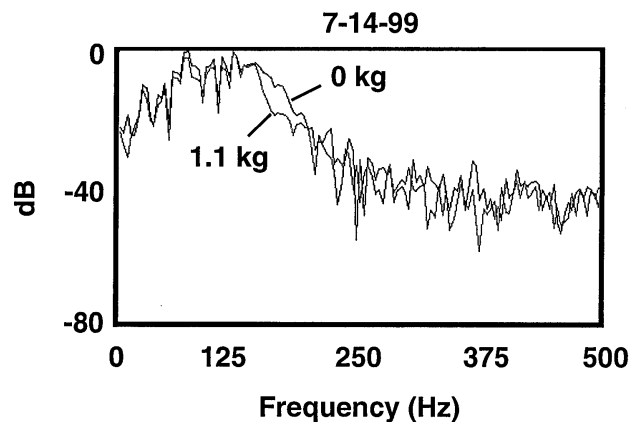

(b)

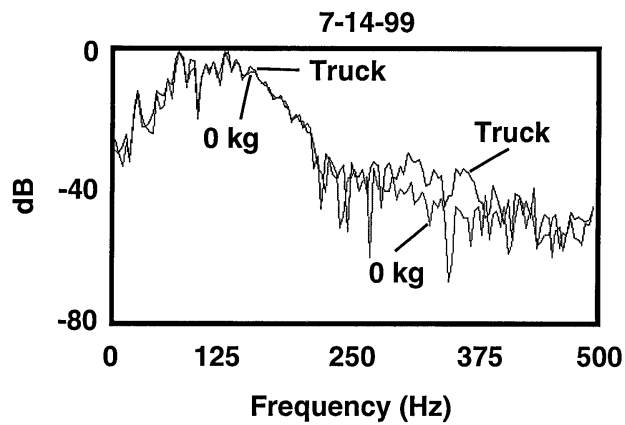

(d)

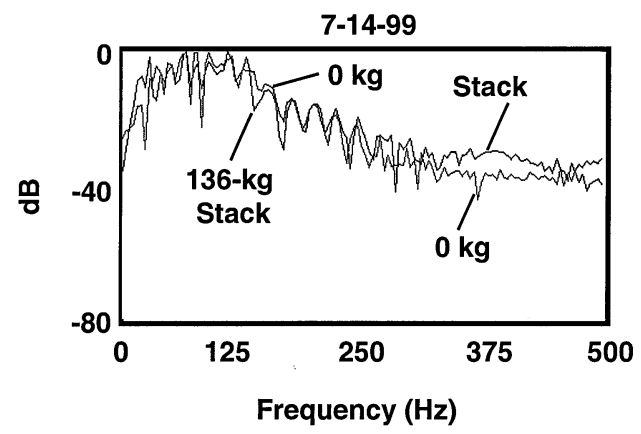

(e)

FIG. 6. Frequency spectra of weighted-geophone traces plotted alongside unweighted-geophone traces from June 30 and August 14 showing (a) and (b) the significant loss of frequency content due to the 1.1-kg weight, and (c), (d), and (e) very little loss due to the mass of the truck and the stack of barbell weights.

decrease amplitude, frequency content, or S/N. Premature first arrivals were the only noticeable effects caused by the mass of the truck (Figures 2,3). These early first breaks possibly are the result of the compaction of the ground around the geophone by the weight of the truck. The compaction may have increased the velocity of the surface layer enough to enable the wave to arrive approximately $1 \mathrm{~ms}$ earlier.

The traces from the most heavily weighted geophones displayed only early first arrivals with little loss of amplitude, frequency, or $\mathrm{S} / \mathrm{N}$; thus, the use of a massive geophone did not cause a critical loss of signal. As a result, an automatic geophone-planting device could be massive without significantly diminishing shallow seismic data quality.

\section{ACKNOWLEDGMENTS}

This work was supported in part by the National Science Foundation Grant EAR97061218, by the U.S. Department of
Energy contract \#DE-FG07-97ER14826, and by a University of Kansas Undergraduate Research Award.

\section{REFERENCES}

Drijkoningen, G. G., 2000, The usefulness of geophone groundcoupling experiments to seismic data: Geophysics, $\mathbf{6 5}, 1780-1787$.

Hoover, G. M., and O'Brien, J. T., 1980, The influence of the planted geophone on seismic land data: Geophysics, 45, 1239-1253.

Jefferson, R. D., Steeples, D. W., Black, R. A., and Carr, T., 1998, Effects of soil-moisture content on shallow seismic data: Geophysics, 63, 1357-1362.

Keiswetter, D., and Steeples, D. W., 1995, A field investigation of source parameters for the sledgehammer: Geophysics, 60, 1051-1057.

Krohn, C. E., 1984, Geophone ground coupling: Geophysics, 49, 722 731.

O'Connor, H. G., 1960, Geology and ground-water resources of Douglas County, Kansas: Kansas Geol Survey Bull. 148.

Steeples, D. W., Baker, G. S., and Schmeissner, C., 1999, Towards the autojuggie: Planting 72 geophones in 2 seconds: Geophys. Res. Lett. 26, $1085-1088$.

Wolf, A., 1944, The equation of motion of a geophone on the surface of an elastic earth: Geophysics, 9, 29-35. 